\title{
Blue Nevus With Rosettes on Polarized Light Dermoscopy
}

\author{
Corina Isabel Salas-Callo, ${ }^{1}$ Josep Riera-Monroig, ${ }^{1}$ Sebastian Podlipnik, ${ }^{1,2}$ Susana Puig ${ }^{1,2,3}$
}

\author{
1 Dermatology Department, Melanoma Unit, Hospital Clínic of Barcelona, Barcelona University, Spain \\ 2 Institut d'Investigacions Biomediques August Pi I Sunyer (IDIBAPS), Barcelona, Spain \\ 3 Biomedical Research Networking Center on Rare Diseases (CIBERER), ISCIII, Barcelona, Spain
}

Key words: blue nevus, rosettes, dermoscopy, polarized light dermoscopy, shiny white structures

Citation: Salas-Callo CI, Riera-Monroig J, Podlipnik S, Puig S. Blue nevus with rosettes on polarized light dermoscopy. Dermatol Pract Concept. 2020;10(1):e2020017. DOI: https://doi.org/10.5826/dpc.1001a17

Accepted: September 21, 2019; Published: December 31, 2019

Copyright: (2019 Salas-Callo et al. This is an open-access article distributed under the terms of the Creative Commons Attribution License, which permits unrestricted use, distribution, and reproduction in any medium, provided the original author and source are credited.

Funding: None.

Competing interests: The authors have no conflicts of interest to disclose.

Authorship: All authors have contributed significantly to this publication.

Corresponding author: Susana Puig, MD, Melanoma Unit, Dermatology Department, Hospital Clinic Barcelona, Villarroel 170. 08036, Barcelona, Spain. Email: susipuig@gmail.com; spuig@clinic.cat

\section{Introduction}

Rosettes are shiny white structures consisting of 4 white dots, similar to a 4-leaf clover. In dermoscopy, they can be seen with polarized light because of an optical phenomenon. Small rosettes (0.1-0.2 mm) are associated with the presence of concentric keratin at the infundibular level of the annex openings, the largest $(0.3-0.5 \mathrm{~mm})$ with perifollicular concentric fibrosis. When multiple rosettes are present, they are usually oriented at the same angle. They have been reported mainly related to actinic keratosis and squamous cell carcinoma. Nevertheless, they are not specific to any condition, as they have been described in a number of inflammatory and tumoral cutaneous lesions, including melanoma [1]. The latter is one of the main differential diagnoses of blue nevi.

Blue nevi are benign proliferations of dendritic melanocytes in the dermis. Clinically they may be acquired or congenital, and they present preferentially in the extremities and face as a blue papule. On dermoscopy, the bluish or steel-blue homogeneous pattern and the absence of other structures are characteristic. However, sometimes it is possible to observe the presence of other structures such as globules, dots, pseudopods, streaks, pigment network, and network-like areas [2]. In these cases, a histopathological study should be performed to rule out melanoma.

\section{Case Presentation}

A 14-year-old male, diagnosed with xeroderma pigmentosum, has come to our center for total body photography every 12 months and digital dermoscopy follow-up every 6 months since he was 6 years old. In this follow-up, an asymptomatic pigmented lesion that had been present since early infancy on the medial region of the dorsum of the right foot was observed and registered. On physical examination it corresponded to a bluish, symmetrical pigmented papule, with a diameter of 5 $\mathrm{mm}$ (Figure 1A). Dermoscopy revealed a homogeneous steelblue pattern with areas of regular gray-blue globules (Figure 1B). In addition, on dermoscopy with polarized light, the lesion showed white rosettes distributed along the length of the whole nevus (Figure 1C). A diagnosis of blue nevus was made, based on the clinical-dermoscopy characteristics. In 
the digital dermoscopy follow-up, this lesion has not presented changes in size or pigment and new structures have not appeared, ruling out the diagnosis of melanoma.

\section{Conclusions}

This description of rosettes in blue nevus draws attention to the importance of the clinical-dermoscopy correlation to establish the diagnosis.

\section{References}

1. Haspeslagh M, Noë M, De Wispelaere I, et al. Rosettes and other white shiny structures in polarized dermoscopy: histological correlate and optical explanation. J Eur Acad Dermatol Venereol. 2016;30(2):311-313.

2. Di Cesare A, Sera F, Gulia A, et al. The spectrum of dermatoscopic patterns in blue nevi. J Am Acad Dermatol. 2012;67(2):199-205.

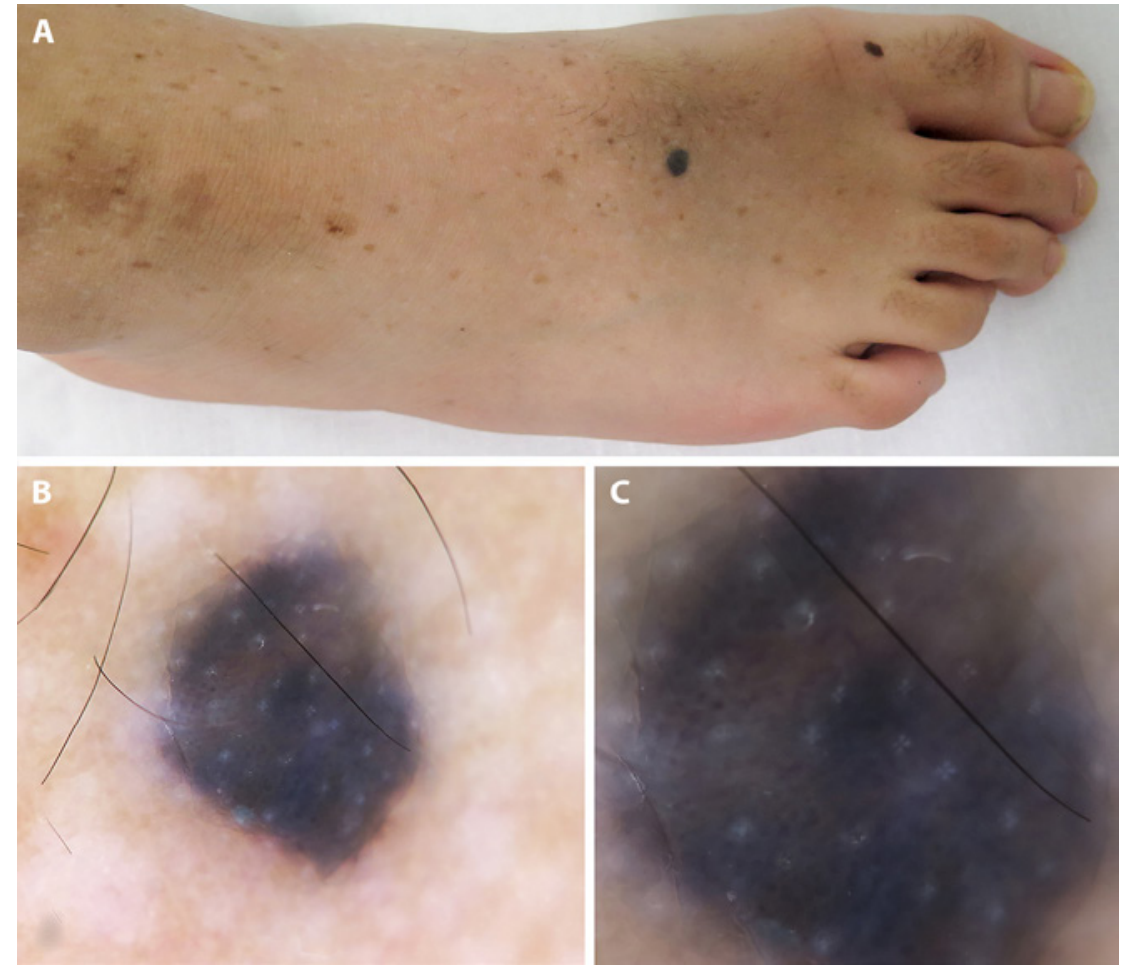

Figure 1. Blue nevus on the dorsum of the right foot. (A) Clinical image. (B) Dermoscopy $(\times 10)$ reveals global steel-blue homogeneous pattern with the presence of multiple white rosettes and the absence of pigment network, globules, and streaks. (C) With greater detail, the orientation of all the rosettes at the same angle. 